\title{
Heat shock protein 27 (HSPB1) suppresses the PDGF-BB-induced migration of osteoblasts
}

\author{
SHINGO KAINUMA ${ }^{1,2}$, HARUHIKO TOKUDA ${ }^{2,3}$, NAOHIRO YAMAMOTO ${ }^{1,2}$, \\ GEN KUROYANAGI $^{1,2}$, KAZUHIKO FUJITA ${ }^{1,2}$, TETSU KAWABATA ${ }^{1,2}$, GO SAKAI ${ }^{1,2}$, \\ RIE MATSUSHIMA-NISHIWAKI $^{2}$, OSAMU KOZAWA ${ }^{2}$ and TAKANOBU OTSUKA ${ }^{1}$

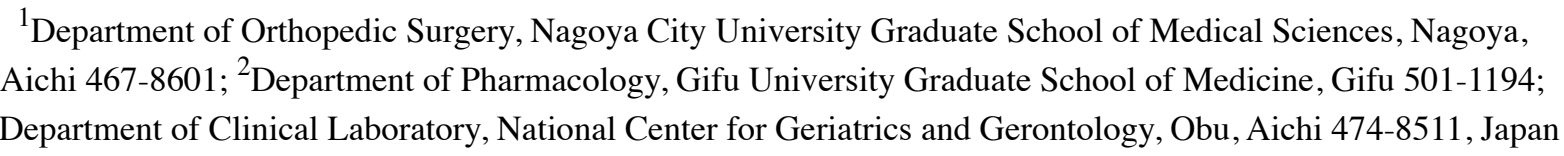

Received March 16, 2017; Accepted August 28, 2017

DOI: $10.3892 / \mathrm{ijmm} .2017 .3119$

\begin{abstract}
Heat shock protein 27 (HSP27/HSPB1), one of the small heat shock proteins, is constitutively expressed in various tissues. HSP27 and its phosphorylation state participate in the regulation of multiple physiological and pathophysiological cell functions. However, the exact roles of HSP27 in osteoblasts remain unclear. In the present study, we investigated the role of HSP27 in the platelet-derived growth factor-BB (PDGF-BB)-stimulated migration of osteoblast-like MC3T3-E1 cells. PDGF-BB by itself barely upregulated the expression of HSP27 protein, but stimulated the phosphorylation of HSP27 in these cells. The PDGF-BB-induced cell migration was significantly downregulated by HSP27 overexpression. The PDGF-BB-induced migrated cell numbers of the wild-type HSP27-overexpressing cells and the phospho-mimic HSP27-overexpressing (3D) cells were less than those of the unphosphorylatable HSP27-overexpressing (3A) cells. PD98059, an inhibitor of MEK1/2, SB203580, an inhibitor of p38 mitogen-activated protein kinase, and SP600125, an inhibitor of stress-activated protein kinase/c-Jun N-terminal kinase (SAPK/JNK) reduced the PDGF-BB-induced migration of these cells, whereas Akt inhibitor or rapamycin, an inhibitor of upstream kinase of p70 S6 kinase (mTOR), barely affected the migration. However, the PDGF-BB-induced phosphorylation of p44/p42 MAPK, p38 MAPK and SAPK/JNK was not affected by HSP27 overexpression. There were no significant differences in the phosphorylation of p44/p42 MAPK, p38 MAP kinase or SAPK/JNK between the 3D cells and the $3 \mathrm{~A}$ cells. These results strongly suggest that HSP27 functions as a negative regulator in the PDGF-BB-stimulated migration
\end{abstract}

Correspondence to: Dr Osamu Kozawa, Department of Pharmacology, Gifu University Graduate School of Medicine, Gifu 501-1194, Japan

E-mail: okozawa@gifu-u.ac.jp

Key words: heat shock protein, heat shock protein 27, platelet-derived growth factor-BB, migration, phosphorylation, osteoblast of osteoblasts, and the suppressive effect is amplified by the phosphorylation state of HSP27.

\section{Introduction}

It is well known that platelet-derived growth factor (PDGF) is a major mitogen for connective tissue cells and certain other cell types (1). The PDGF family consists of four different polypeptide chains encoded by different genes, which have been identified as PDGF-A, PDGF-B and the recently discovered PDGF-C and PDGF-D, and 4 homodimers (PDGF-AA, PDGF-BB, PDGF-CC and PDGF-DD) and a heterodimer (PDGF-AB) (2). To maintain structural bone integrity and mineral homeostasis, bone metabolism is tightly regulated by bone remodeling, which consists mainly of osteoblast bone formation and osteoclastic bone resorption (3). In bone metabolism, PDGF reportedly increases proliferation of osteoblasts and inhibits differentiation (4). As for the fracture healing process, PDGF has a crucial role as a systemic factor, and regulates bone remodeling (5). Additionally, it has been reported that administration of recombinant platelet-derived growth factor-BB (PDGF-BB) accelerates fracture healing in the geriatric osteoporotic rat (6). We previously demonstrated that PDGF-BB stimulates interleukin-6 (IL-6) synthesis via p44/ p42 mitogen-activated protein kinase (MAPK), p38 MAPK and stress-activated protein kinase/c-Jun N-terminal kinase (SAPK/JNK) in osteoblast-like MC3T3-E1 cells (7), and that phosphoinositide 3-kinase (PI3K)/Akt and p70 S6 kinase negatively regulate the synthesis $(7,8)$. It has recently been reported that PDGF-BB induces the migration of human osteoblasts (9). However, the details behind the effect of PDGF effect in osteoblast migration has not been fully clarified.

It is generally recognized that heat shock proteins (HSPs) play pivotal roles as molecular chaperones, which facilitate the refolding of improperly folded proteins induced by stress, or assist in their elimination via chaperone-mediated autophagy or the ubiquitin proteasome system (10). Among them, HSP27 (HSPB1) belongs to the HSPB family, with monomeric molecular masses ranging from 12 to $43 \mathrm{kDa}$, so called small HSPs. HSP27 is ubiquitously expressed in human cells and tissues, and its functions have been clarified in the 
studies of tissues which have higher expression levels such as smooth, skeletal and cardiac muscles (11). Furthermore, it is generally known that post-translational modifications such as phosphorylation regulate the functions of HSP27 (12). HSP27 ordinarily exists as unphosphorylated multioligomers in cells and possesses three serine residues (Ser-15, Ser-78 and Ser-82) and one threonine residue (Thr-143) that can be phosphorylated by related enzymes such as p38 MAPK $(12,13)$. HSP27 oligomerization is mainly regulated by Ser-78 and/or Ser-82 phosphorylation (13). Once HSP27 is phosphorylated, a conformational change occurs from the aggregated form to the dimer $(11,12)$. With regard to HSP27 in osteoblasts, HSP27 is reportedly involved in their differentiation and apoptosis (14). The expression levels of HSP27 in osteoblasts under unstimulated conditions (15) including osteoblast-like MC3T3-E1 cells (16) are quite low. In our previous studies, we demonstrated that various bone remodeling mediators, such as prostaglandin $\mathrm{D}_{2}\left(\mathrm{PGD}_{2}\right)$, endothelin-1, $\mathrm{PGE}_{2}, \mathrm{PGF}_{20}$ and transforming growth factor- $\beta$ (TGF- $\beta$ ), enable HSP27 to be induced in osteoblast-like MC3T3-E1 cells (17-21). In addition, we reported that phosphorylated HSP27 changes its localization from the cytosol to the perinuclear region, and acts as a negative regulator in triiodothyronine-induced osteocalcin synthesis in MC3T3-E1 cells, but has a stimulatory effect on mineralization (22). However, the exact roles of HSP27 especially in the function of osteoblasts including migration remain to be elucidated.

In the present study, we investigated the role of HSP27 in the PDGF-BB-stimulated migration of osteoblast-like MC3T3-E1 cells. We herein showed that HSP27 acts as a negative regulator in PDGF-BB-stimulated cell migration, and the inhibitory effect of phosphorylated HSP27 on the migration is stronger than that of unphosphorylated HSP27.

\section{Materials and methods}

Materials. PDGF-BB and TGF- $\beta$ were both purchased form R\&D Systems, Inc. (Minneapolis, MN, USA). HSP27 antibodies used to detect the expression of HSP27, glyceraldehyde 3-phosphate dehydrogenase (GAPDH) antibodies and deguelin, were obtained from Santa Cruz Biotechnology, Inc. (Santa Cruz, CA, USA). HSP27 antibodies used to confirm the transfection with the WT HSP27 vector, and phospho-specific (p)-HSP27 (Ser-82) antibodies were purchased from Enzo Life Sciences, Inc. (Famingdale, NY, USA). PD98059, SB203580, SP600125, Akt inhibitor and rapamycin were all obtained from Calbiochem-Novabiochem (La Jolla, CA, USA). p-p44/p42 MAPK, p-p38 MAPK and p-SAPK/JNK antibodies were all purchased from Cell Signaling Technology, Inc. (Beverly, MA, USA). WT HSP27 and mutant human HSP27 subcloned into the pcDNA3.1(+) mammalian expression vector were kindly provided by Dr. C. Schafer (Klinikum Grosshadern, Ludwig Maximilians University, Munich, Germany). Regarding mutant HSP27 vectors, three serine residues (Ser-15, 78 and 82) of the HSP27 cDNAs had been mutated to alanine (3A) to prevent the phosphorylation of HSP27, or mutated to aspartic acid (3D) to imitate the phosphorylated HSP27 form, as previously described (23). The eukaryotic expression vector, pcDNA3.1(+) was obtained from Life Technologies (Carlsbad, CA, USA). A BCA protein assay kit was purchased from
Thermo Fisher Scientific Inc. (Waltham, MA, USA). An ECL western blotting detection system was obtained from GE Healthcare UK Ltd. (Buckinghamshire, UK). Other materials and chemicals were obtained from commercial sources. PD98059, SB203580, SP600125, Akt inhibitor, deguelin and rapamycin were dissolved in dimethyl sulfoxide (DMSO). The maximum concentration of DMSO was $0.3 \%$, which did not affect the cell migration assay.

Cell culture. Cloned osteoblast-like MC3T3-E1 cells, which were originally derived from newborn mouse calvaria (24) were maintained as previously described (25). In brief, the cells were maintained in $\alpha$-minimum essential medium ( $\alpha$-MEM) containing 10\% fetal bovine serum (FBS) in a humidified $95 \%$ air, $5 \% \mathrm{CO}_{2}$ atmosphere at $37^{\circ} \mathrm{C}$. The cells

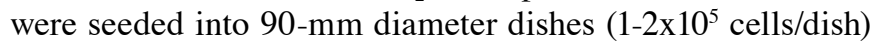
in $\alpha$-MEM containing $10 \%$ FBS. After 4 days, the medium was exchanged for $\alpha$-MEM containing $0.3 \%$ FBS. Thereafter, the cells were cultured for $6 \mathrm{~h}$ and then used for the cell migration assay.

Transient transfections. For transient transfections, the MC3T3-E1 cells were seeded into 90-mm diameter dishes (5-15x $10^{4}$ cells/dish) in $\alpha$-MEM containing $10 \%$ FBS. After 4 days, the medium was removed, and the cells were washed with $10 \mathrm{ml}$ of $\alpha$-MEM medium without FBS. The cultured cells were then transfected with $6.6 \mu \mathrm{g}$ of the WT, control (empty), mutant $3 \mathrm{~A}$ or $3 \mathrm{D}$ HSP27 plasmids pcDNA3.1(+) vector using the UniFector transfection reagent (B-Bridge International, Inc., Mountain View, CA, USA) in $5.6 \mathrm{ml}$ of $\alpha$-MEM medium without FBS. Five hours after transfection, $6.6 \mathrm{ml}$ of medium with $\alpha$-MEM containing $0.6 \%$ FBS was added. At 3 days after transfection, the medium was exchanged to $\alpha$-MEM containing $0.3 \%$ FBS for the experiments.

Cell migration assay. Cell migration assay was performed as previously described in the method of Karagiosis et al (26) using Boyden chamber [polycarbonate membrane with 8- $\mu \mathrm{m}$ pores (Transwell ${ }^{\circledR}$; Corning Costar, Cambridge, MA, USA)]. Briefly, the cultured cells were trypsinized, and seeded (5-10x $10^{4}$ cells/well) onto the upper chamber in $\alpha$-MEM containing $0.3 \%$ FBS. PDGF-BB $(3 \mathrm{ng} / \mathrm{ml})$ was added to the lower chamber, and the cells were incubated for $16 \mathrm{~h}$ at $37^{\circ} \mathrm{C}$. After mechanical removal of the cells on the upper surface of the membrane, the migrated cells adherent to the underside of the membrane were fixed with $4 \%$ paraformaldehyde and stained with 4',6-diamidino-2-phenylindole (DAPI) solution. The number of stained cells was counted using fluorescence microscopy from three randomly chosen high power fields per well at $\mathrm{x} 20$ magnification. When indicated, the cells were used after pretreatment with PD98059, SB203580, SP600125, Akt inhibitor, deguelin or rapamycin in the lower chamber for $60 \mathrm{~min}$

Western blot analysis. The cultured cells or transfected cells were stimulated by $30 \mathrm{ng} / \mathrm{ml}$ of PDGF-BB or $5 \mathrm{ng} / \mathrm{ml}$ of TGF- $\beta$ in $\alpha$-MEM containing $0.3 \%$ FBS for the indicated periods. The cells were washed twice in phosphate-buffered saline (PBS) and then lysed, homogenized and sonicated in a lysis buffer: $62.5 \mathrm{mM}$ Tris- $\mathrm{HCl}, \mathrm{pH}$ 6.8, 2\% sodium dodecyl 

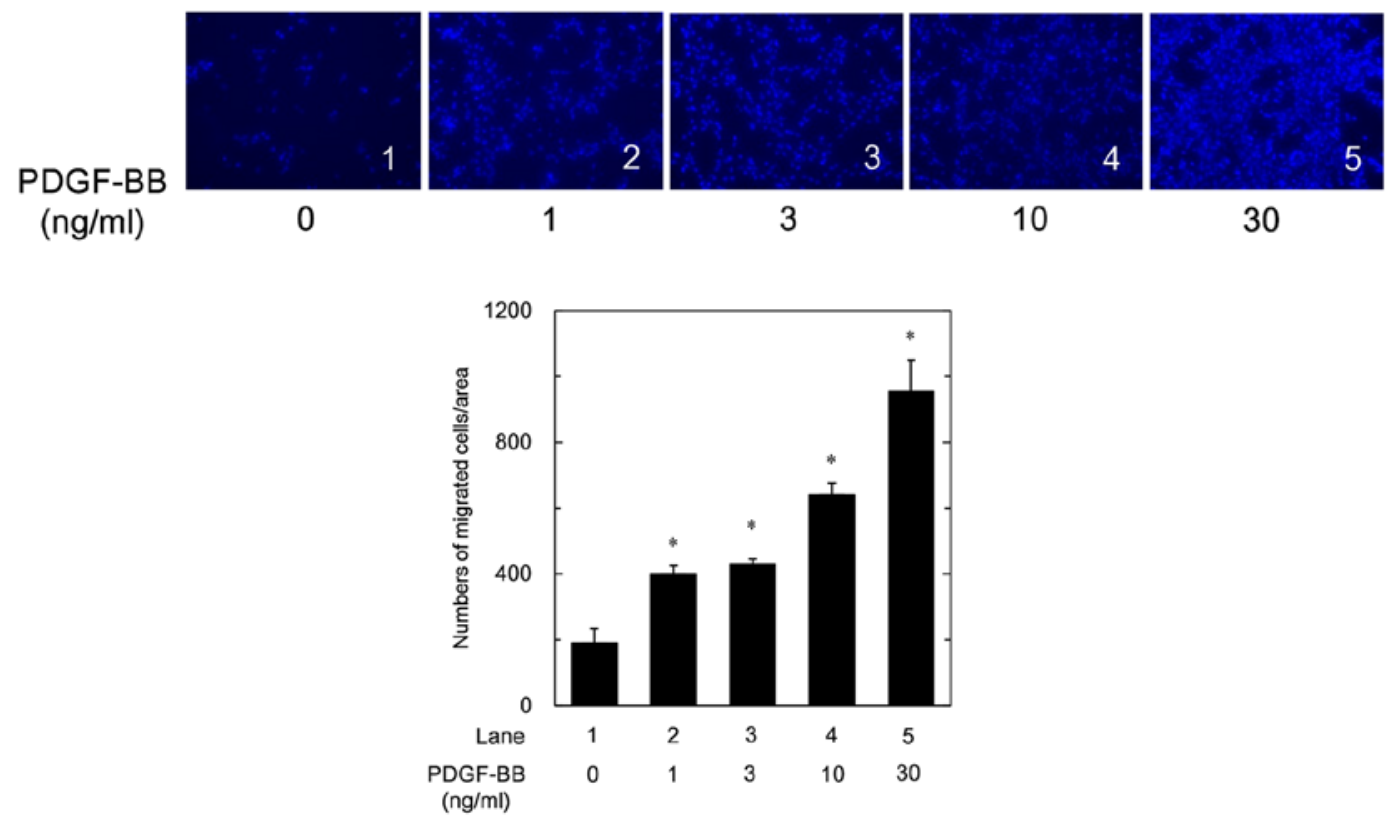

Figure 1. Effect of platelet-derived growth factor-BB (PDGF-BB) on the migration of MC3T3-E1 cells. The cultured cells were stimulated by various doses of PDGF-BB for $16 \mathrm{~h}$. The migrated cells were stained with DAPI for visualization of the nucleus (blue signal). Fluorescence microscopy (upper panel) and the number of migrated cells (lower panel). Each value represents the mean \pm SEM of triplicate determinations from three independent cell preparations. ${ }^{*} \mathrm{P}<0.05$ compared to the value of the control cells without PDGF-BB stimulation.

sulfate (SDS), $50 \mathrm{mM}$ dithiothreitol and $10 \%$ glycerol. The protein concentration of each sample was measured by a BCA protein assay kit. SDS-polyacrylamide gel electrophoresis (PAGE) was performed by the method described by Laemmli (27) on $10 \%$ polyacrylamide gels. The samples from the transfected cell cultures to be quantitatively compared by the western blot analysis were run on the same gel. The protein was fractionated and transferred onto an Immun-Blot polyvinylidene difluoride membrane (Bio-Rad Laboratories, Inc., Hercules, CA, USA). The membranes were blocked with $5 \%$ fat-free dry milk in Tris-buffered saline-Tween (TBS-T; $20 \mathrm{mM}$ Tris-HCl, pH 7.6, $137 \mathrm{mM} \mathrm{NaCl}, 0.1 \%$ Tween-20) for $1 \mathrm{~h}$ before incubation with the primary antibodies. Western blot analysis was performed as previously described (28) using the indicated primary antibodies with the appropriate secondary antibodies. Immunoreactive bands were visualized on X-ray film by means of the ECL western blotting detection system.

Determinations. The densitometric analysis was carried out using image analysis software (ImageJ version 1.49; National Institutes of Health, Bethesda, MD, USA). The background-subtracted signal intensity for each protein and phosphorylation was normalized to the respective intensity of GAPDH, and plotted as the fold increase in comparison to the control cells.

Statistical analysis. All data are presented as the mean \pm standard error of the mean (SEM) of triplicate determinations from three independent cell preparations. Statistical analysis were performed using the Bonferroni method for multiple comparisons between pairs, and a P-value $<0.05$ was considered to be significant.

\section{Results}

Effects of PDGF-BB on the migration, HSP27 induction and HSP27 phosphorylation in MC3T3-E1 cells. It has been reported that $\mathrm{PDGF}-\mathrm{BB}$ induces the migration of human osteoblasts (9). We found that PDGF-BB truly stimulated the migration of osteoblast-like MC3T3-E1 cells in a dose-dependent manner in the range between 1 and $30 \mathrm{ng} / \mathrm{ml}$ (Fig. 1). In our previous studies $(16,29,30)$, we demonstrated that osteoblast-like MC3T3-E1 cells normally have low levels of HSP27, and HSP27 expression is induced by sphingosine 1-phosphate, basic fibroblast growth factor or TGF- $\beta$. We confirmed that HSP27 levels without stimulation were low in these cells. PDGF-BB barely induced the expression of HSP27 up to $12 \mathrm{~h}$ in comparison to TGF- $\beta$ (Fig. $2 \mathrm{~A}$ ).

We previously established WT HSP27-overexpressing MC3T3-E1 cells transfected with the WT HSP27 vector (31). We confirmed that the protein expression levels of HSP27 were clearly detectable in the WT HSP27 vector-transfected MC3T3-E1 cells, in comparison with the control empty vector-transfected cells (Fig. 2B). We next examined the effect of PDGF-BB on the phosphorylation of HSP27 in the WT HSP27 vector-transfected MC3T3-E1 cells. We confirmed that the phosphorylation of HSP27 was barely detected before the PDGF-BB stimulation in the HSP27overexpressing MC3T3-E1 cells, but found that PDGF-BB markedly stimulated the phosphorylation of HSP27 (Fig. 2C). The effect of PDGF-BB on the phosphorylation of HSP27 was detected from 10 to $30 \mathrm{~min}$ after the stimulation. We confirmed that PDGF-BB markedly induced the phosphorylation of HSP27 in the HSP27-overexpressing cells at $10 \mathrm{~min}$ after the stimulation in comparison with that without PDGF-BB stimulation (Fig. 2D). 
A

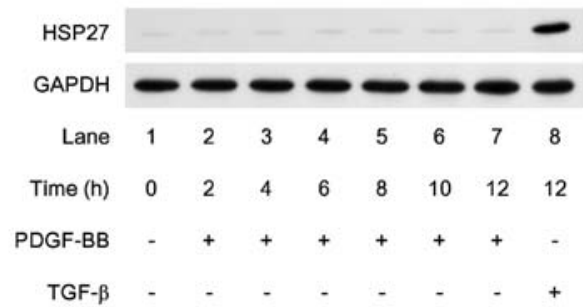

C

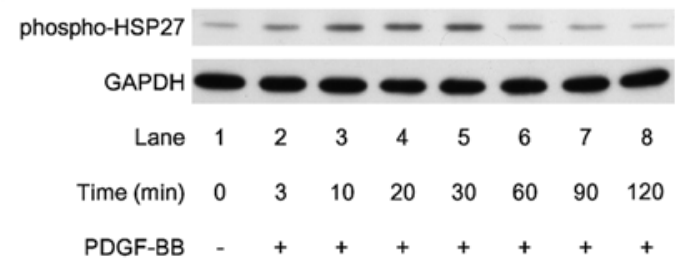

B

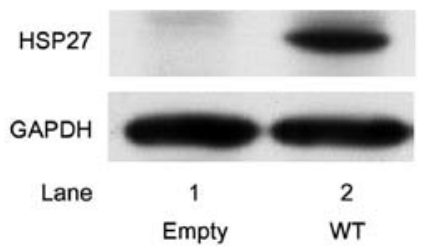

D

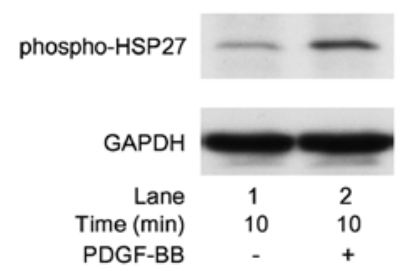

Figure 2. Effects of platelet-derived growth factor-BB (PDGF-BB) on the induction and the phosphorylation of heat shock protein 27 (HSP27) in MC3T3-E1 cells. (A) The cultured cells were stimulated with $30 \mathrm{ng} / \mathrm{ml}$ of PDGF-BB, $5 \mathrm{ng} / \mathrm{ml}$ of transforming growth factor- $\beta$ (TGF- $\beta$ ) or vehicle for the indicated time periods. (B) The cultured cells were transiently transfected with the control empty (empty) vector or wild-type (WT) HSP27 vector, and were subsequently cultured for 3 days. (C and D) The cultured cells were transiently transfected with the WT HSP27 vector, and were subsequently cultured for 3 days. (C) The transfected cells were then stimulated with $30 \mathrm{ng} / \mathrm{ml}$ of PDGF-BB for the indicated periods. (D) The transfected cells were then stimulated with $30 \mathrm{ng} / \mathrm{ml}$ of PDGF-BB or vehicle for $10 \mathrm{~min}$. The cell extracts were subjected to SDS-PAGE with subsequent western blot analysis using antibodies against HSP27 (A and B) and phospho-specific HSP27 (C and D) or GAPDH.
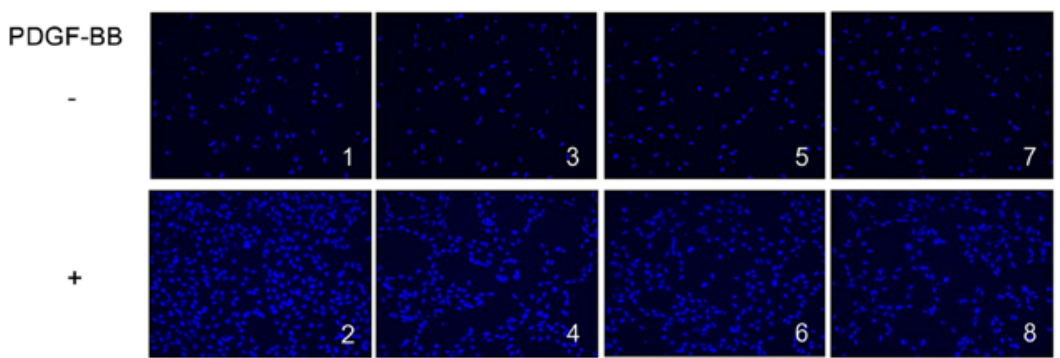

HSP27

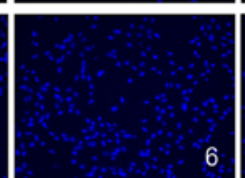

$3 \mathrm{~A}$

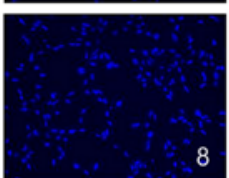

$3 \mathrm{D}$

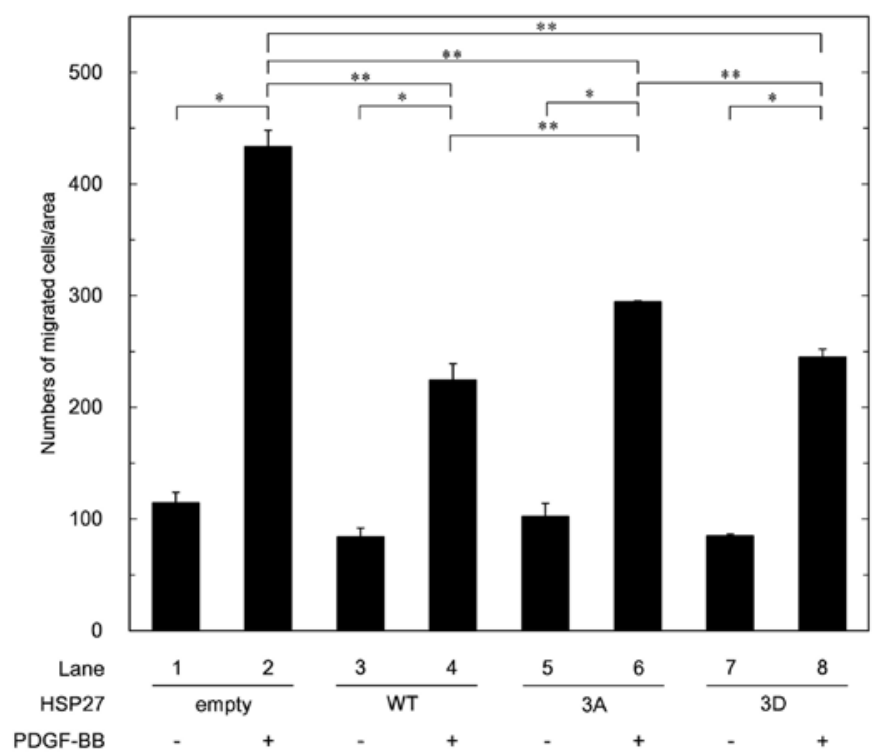

Figure 3. Effect of platelet-derived growth factor (PDGF) on the migration of wild-type (WT) heat shock protein 27 (HSP27)- or mutant HSP27-transfected MC3T3-E1 cells. The cultured cells were transiently transfected with the control empty (empty), the WT HSP27, the unphosphorylatable (3A) HSP27 or the phospho-mimic (3D) HSP27 vectors, and were subsequently cultured for 3 days. The transfected cells were then stimulated with 3 ng/ml of PDGF-BB or vehicle for $16 \mathrm{~h}$. The migrated cells were stained with DAPI for nuclear visualization (blue signal). Fluorescence microscopy (upper panel) and the number of migrated cells (lower panel). Each value represents the mean \pm SEM of triplicate determinations from three independent cell preparations. ${ }^{*}<0.05$ compared to the value without PDGF-BB stimulation in each; ${ }^{* *} \mathrm{P}<0.05$ compared to the value with PDGF-BB stimulation in each. 
A
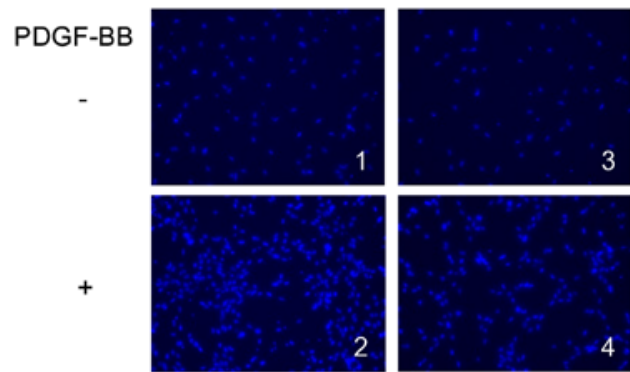

PD98059

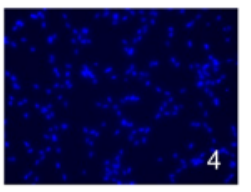

$+$

B
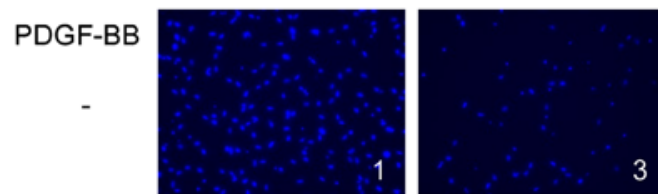

2
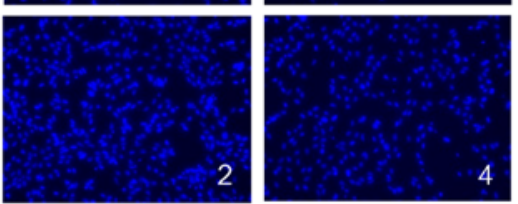

SB203580

$+$
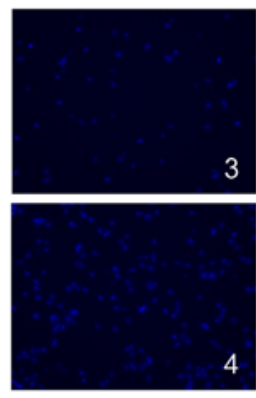

SP600125
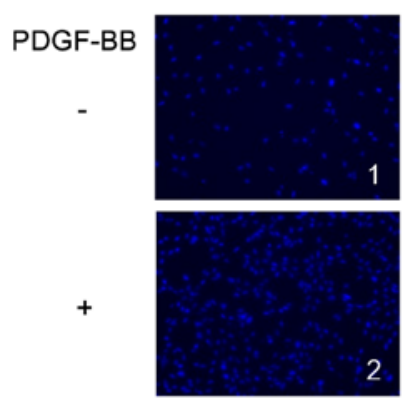

$-$
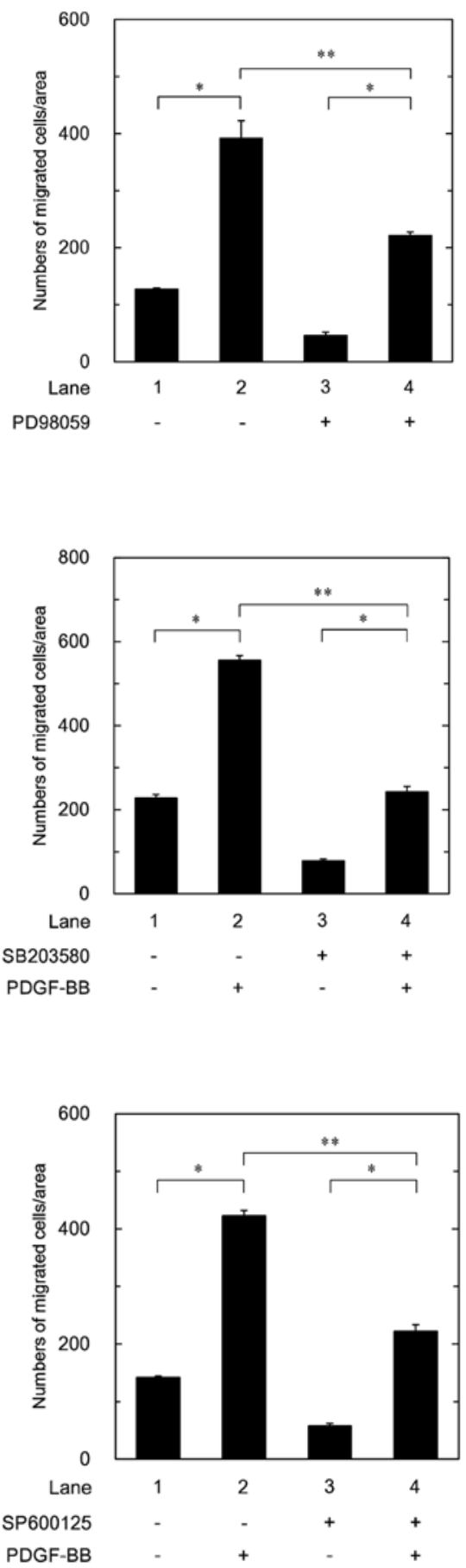

Figure 4. Effects of PD98059, SB203580 or SP600125 on the platelet-derived growth factor-BB (PDGF-BB)-stimulated migration of MC3T3-E1 cells. The cultured cells were pretreated with (A) $50 \mu \mathrm{M}$ of PD98059, (B) $30 \mu \mathrm{M}$ of SB203580, (C) $10 \mu \mathrm{M}$ of SP600125 or vehicle for 60 min, and then stimulated with 3 ng/ $\mathrm{ml}$ of PDGF-BB or vehicle for $16 \mathrm{~h}$. The migrated cells were stained with DAPI for nuclear visualization (blue signal). Fluorescence microscopy (left panel) and the number of migrated cells (right panel). Each value represents the mean \pm SEM of triplicate determinations from three independent cell preparations. ${ }^{*} \mathrm{P}<0.05$ compared to the value without $\mathrm{PDGF}-\mathrm{BB}$ stimulation in each; ${ }^{* *} \mathrm{P}<0.05$ compared to the value with $\mathrm{PDGF}-\mathrm{BB}$ stimulation in each.

Effects of HSP27 overexpression and the status of HSP27 phosphorylation on the PDGF-BB-induced migration of $M C 3 T 3-E 1$ cells. It is currently recognized that the functions of HSP27 are modulated by phosphorylation, resulting from its conformational changes through dimerization of multioligomers (10). We previously established two mutant HSP27-transfected MC3T3-E1 cell lines, in which serine residues (Ser-15, Ser-78 and Ser-82) were mutated into alanine (3A) to prevent the phosphorylation or were mutated into aspartic acid (3D) to imitate the phosphorylated form, and reported that the phosphorylation levels of HSP27 in the 3D-transfected cells were clearly greater than those in the 3A-transfected cells (22). We investigated the effects of PDGF-BB on the migration of control empty vector-transfected cells which represent normal MC3T3-E1 cells, WT HSP27-overexpressing cells, 3A cells and $3 \mathrm{D}$ cells on the same day. There were no differences in 
A
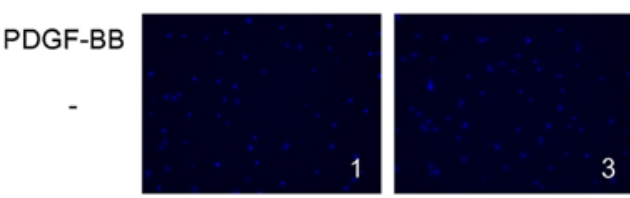

2

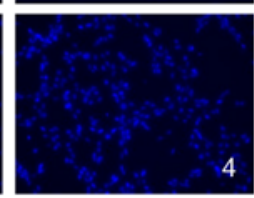

Akt inhibitor

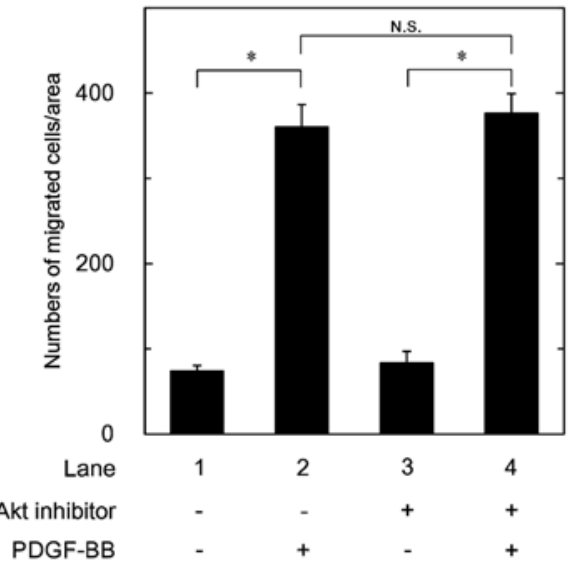

B

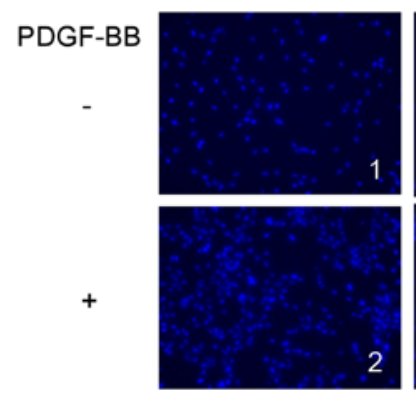

Rapamycin

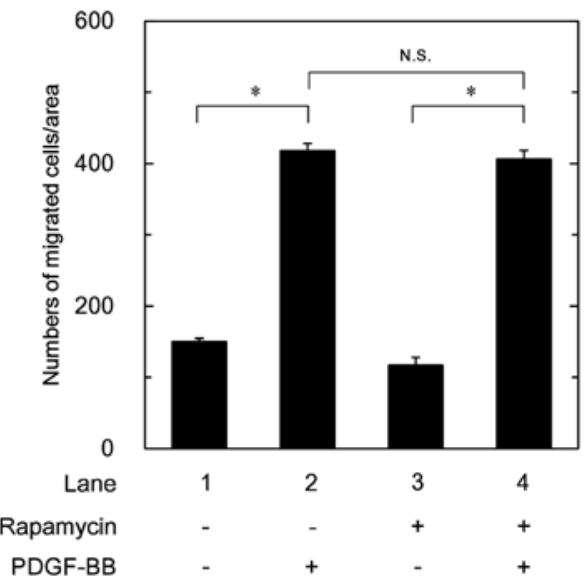

Figure 5. Effects of Akt inhibitor or rapamycin on the platelet-derived growth factor-BB (PDGF-BB)-stimulated migration of MC3T3-E1 cells. The cultured cells were pretreated with (A) $30 \mu \mathrm{M}$ of Akt inhibitor, (B) $50 \mathrm{ng} / \mathrm{ml}$ of rapamycin or vehicle for $60 \mathrm{~min}$, and then stimulated with $3 \mathrm{ng} / \mathrm{ml}$ of PDGF-BB or vehicle for $16 \mathrm{~h}$. The migrated cells were stained with DAPI for nuclear visualization (blue signal). Fluorescent microscopy (upper panel) and the number of migrated cells (lower panel). Each value represents the mean \pm SEM of triplicate determinations from three independent cell preparations. ${ }^{*}<0.05$ compared to the value without PDGF-BB stimulation in each. N.S. designates no significant difference between the indicated pairs.

the migrated cell numbers among the different cell groups without PDGF-BB stimulation. In comparison with the control empty vector-transfected cells, the PDGF-BB-induced migration in the WT HSP27-overexpressing cells was significantly reduced. Although the difference in the PDGF-BB-induced migrated cell numbers between the $3 \mathrm{D}$ cells and the $3 \mathrm{~A}$ cells appeared subtle, the migrated numbers of the $3 \mathrm{D}$ cells were significantly reduced compared with those of the $3 \mathrm{~A}$ cells. In addition, the PDGF-BB-induced migrated cell numbers of the WT HSP27-overexpressing cells were less than those of the 3A cells (Fig. 3).

Effects of PD98059, SB203580 or SP600125 on the PDGF-BB-stimulated migration of MC3T3-E1 cells. Regarding the intracellular signaling of PDGF-BB in osteoblasts, we previously demonstrated that $\mathrm{p} 44 / \mathrm{p} 42 \mathrm{MAPK}, \mathrm{p} 38 \mathrm{MAPK}$ and SAPK/JNK function as positive regulators in the PDGF-BBstimulated IL-6 synthesis in osteoblast-like MC3T3-E1 cells, whereas the PI3K/Akt and p70 S6 kinase pathways negatively regulate the IL-6 synthesis $(7,8)$. In order to investigate the roles of three MAPKs in the PDGF-BB-stimulated cell migration, we examined the effects of PD98059, an inhibitor of the upstream kinase activating p44/p42 MAPK (MEK1/2) (32), SB203580, an inhibitor of p38 MAPK (33), or SP600125, an inhibitor of SAPK/JNK (34), on the cell migration. PD98059, SB203580 and SP600125 significantly reduced the PDGF-BBstimulated migration of the MC3T3-E1 cells (Fig. 4A-C).
Effects of Akt inhibitor or rapamycin on the PDGF-BB-stimulated migration of MC3T3-E1 cells. In order to clarify the role of PI3K/Akt or p70 S6 kinase in the PDGF-BB-stimulated migration of MC3T3-E1 cells, we next examined the effects of Akt inhibitor, $1_{\mathrm{L}}-6$-hydroxymethyl-chiroinositol 2-(R)-2-O-methyl-3-O-octadecylcarbonate (35) and rapamycin, an inhibitor of upstream kinase of p70 S6 kinase (mTOR) (36), on the cell migration. The Akt inhibitor failed to affect the PDGF-BB-stimulated migration of MC3T3-E1 cells (Fig. 5A). In addition, we found that deguelin, another Akt inhibitor (37), did not suppress the PDGF-BB-induced cell migration (data not shown). On the other hand, rapamycin barely affected the PDGF-BBstimulated migration of MC3T3-E1 cells (Fig. 5B).

Effects of HSP27 overexpression on the PDGF-BB-induced phosphorylation of $44 / p 42$ MAPK, p38 MAPK or SAPK/JNK in MC3T3-E1 cells. In order to clarify whether HSP27 affects PDGF-BB-stimulated MC3T3-E1 cell migration through the activation of p44/p42 MAPK, p38 MAPK or SAPK/JNK, we examined the effect of PDGF-BB on the phosphorylation of p44/p42 MAPK, p38 MAPK or SAPK/JNK in the WT HSP27 vector-transfected MC3T3-E1 cells and the control empty vector-transfected cells. However, there were no significant differences in the phosphorylated levels of p44/p42 MAPK, p38 MAPK or SAPK/JNK between the WT HSP27-overexpressing cells and the control cells (Fig. 6A-C). 
A

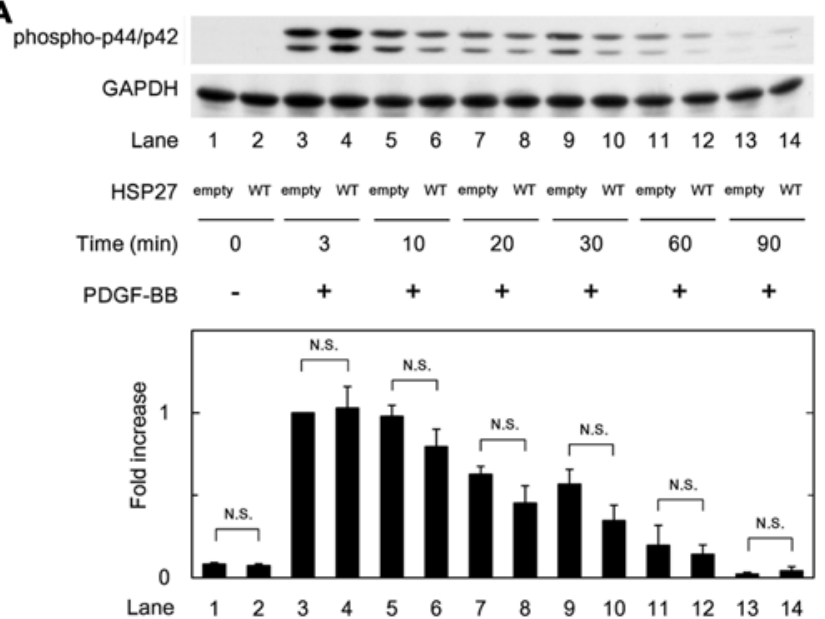

B

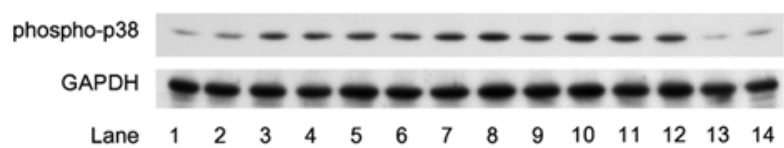

HSP27 emply WT empty WT emply WT empty wT empty WT emply wT emply WT
Time (min)

PDGF-BB - $++\quad+\quad+\quad+$

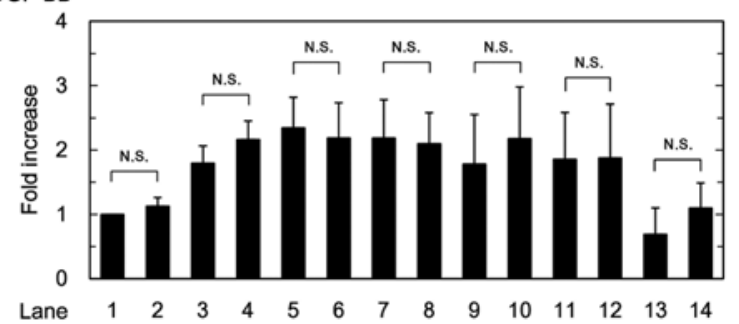

Chospho-SAPKIJNK $-\cdots-\cdots_{-}$

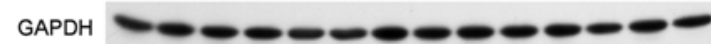

$\begin{array}{lllllllllllllll}\text { Lane } & 1 & 2 & 3 & 4 & 5 & 6 & 7 & 8 & 9 & 10 & 11 & 12 & 13 & 14\end{array}$

HSP27 empty WT empty WT empty WT empty WT empty WT empty WT empty WT

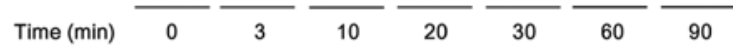

PDGF-BB $\quad-\quad+\quad+\quad+\quad+\quad+\quad+\quad+\quad+\quad+$

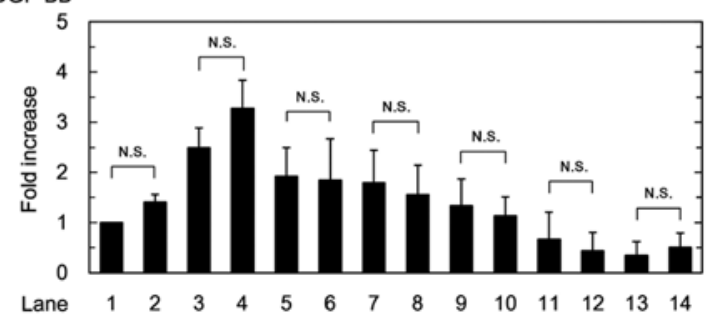

Figure 6. Effects of heat shock protein 27 (HSP27) overexpression on the phosphorylation of p44/p42 mitogen-activated protein kinase (MAPK), p38 MAPK or stress-activated protein kinase/c-Jun N-terminal kinase (SAPK/JNK) in MC3T3-E1 cells. The cultured cells were transiently transfected with the control empty vector (empty) or the wild-type (WT) HSP27 vectors. The transfected cells were stimulated with $30 \mathrm{ng} / \mathrm{ml}$ of PDGF-BB or vehicle for the indicated periods. The cell extracts were then subjected to SDS-PAGE with subsequent western blot analysis using antibodies against (A) phospho-specific p44/p42 MAPK, (B) phospho-specific p38 MAPK, (C) phospho-specific SAPK/JNK or GAPDH. The histogram shows quantitative representations of the levels of PDGF-BB-induced phosphorylation of (A) p44/p42 MAPK, (B) p38 MAPK and (C) SAPK/JNK obtained after normalization with respect to GAPDH from laser densitometric analysis. Each value represents the mean \pm SEM of triplicate determinations from three independent cell preparations. N.S. designates no significant difference between the indicated pairs.

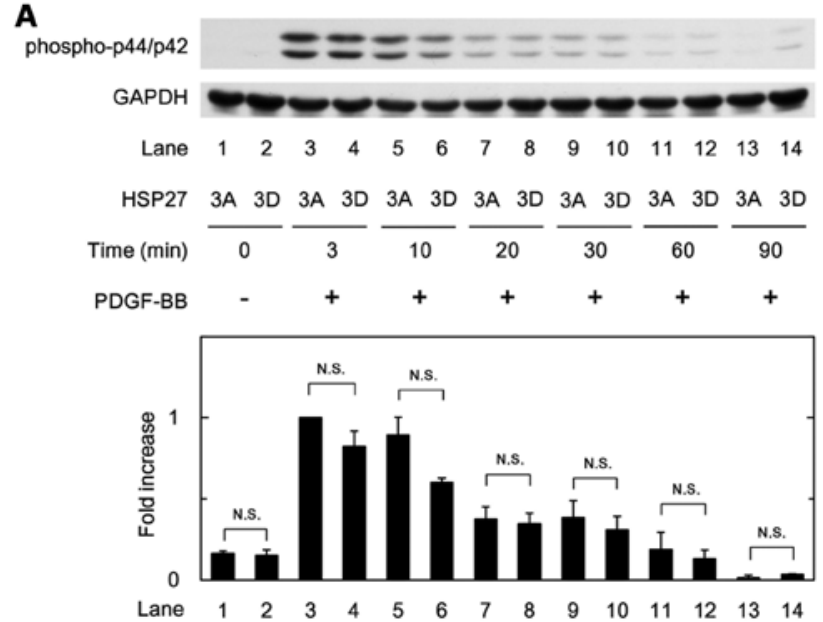

B

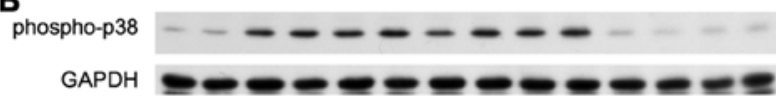

$\begin{array}{lllllllllllllll}\text { Lane } & 1 & 2 & 3 & 4 & 5 & 6 & 7 & 8 & 9 & 10 & 11 & 12 & 13 & 14\end{array}$

HSP27 3A 3D 3A 3D 3A 3D $3 A$ 3D $3 A$ 3D $3 A$ 3D $3 A$ 3D Time (min) $\frac{}{0}-\frac{3}{3} \frac{}{10} \frac{}{20} \frac{}{30} \frac{}{60} \frac{}{90}$ PDGF-BB $\quad-\quad+\quad+\quad+\quad+\quad+\quad+$

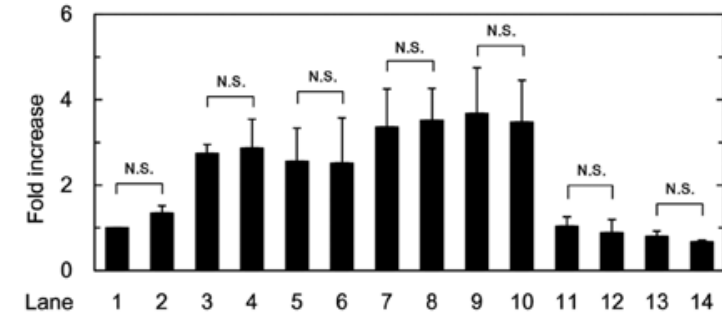

C

phospho-SAPKIJNK $-\cdots-\cdots$

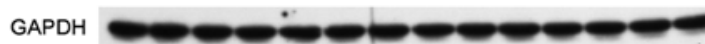

$\begin{array}{lllllllllllllll}\text { Lane } & 1 & 2 & 3 & 4 & 5 & 6 & 7 & 8 & 9 & 10 & 11 & 12 & 13 & 14\end{array}$

$\begin{array}{lllllllllllllll}\text { HSP27 } & 3 A & 3 D & 3 A & 3 D & 3 A & 3 D & 3 A & 3 D & 3 A & 3 D & 3 A & 3 D & 3 A & 3 D\end{array}$

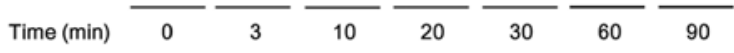
PDGF-BB $\quad-\quad+\quad+\quad+\quad+\quad+\quad+$

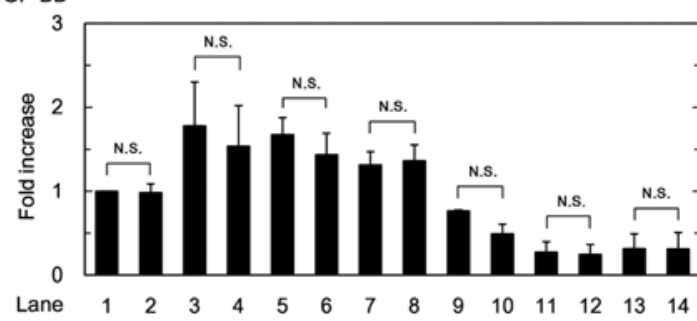

Figure 7. Effects of heat shock protein 27 (HSP27) phosphorylation status on the phosphorylation of p44/p42 mitogen-activated protein kinase (MAPK), p38 MAPK or stress-activated protein kinase/c-Jun N-terminal kinase (SAPK/JNK) in MC3T3-E1 cells. The cultured cells were transiently transfected with either the phospho-mimic (3D) or the unphosphorylatable (3A) HSP27 vectors. The transfected cells were then stimulated with $30 \mathrm{ng} / \mathrm{ml}$ of PDGF-BB or vehicle for the indicated periods. The cell extracts were then subjected to SDS-PAGE with subsequent western blot analysis using antibodies against (A) phospho-specific p44/p42 MAPK, (B) phospho-specific p38 MAPK, (C) phospho-specific SAPK/JNK or GAPDH. The histogram shows quantitative representations of the levels of PDGF-BB-induced phosphorylation of (A) p44/p42 MAPK, (B) p38 MAPK and (C) SAPK/JNK obtained after normalization with respect to GAPDH from laser densitometric analysis. Each value represents the mean \pm SEM of triplicate determinations from three independent cell preparations. N.S. designates no significant difference between the indicated pairs. 
We further examined the effect of PDGF-BB on the phosphorylation of p44/p42 MAPK, p38 MAPK or SAPK/ JNK in the 3D and 3A cells. There were no significant differences in their phosphorylated levels between the $3 \mathrm{~A}$ and $3 \mathrm{D}$ cells (Fig. 7A-C).

\section{Discussion}

We conducted the present study to clarify whether HSP27 (HSPB1), one of the low-molecular-weight HSPs, is implicated in the migration of osteoblast-like MC3T3-E1 cells. Since PDGF-BB, a potent mitogen of connective tissue cells (1), reportedly induces migration of human osteoblasts, we confirmed that PDGF-BB truly stimulated the migration of osteoblast-like MC3T3-E1 cells. Although HSP27 is ubiquitously expressed in human cells and tissues such as skeletal, smooth and cardiac muscles, we herein confirmed that the expression levels of HSP27 without stimulation are quite low in MC3T3-E1 cells as we previously reported (16). We also found that PDGF-BB barely induced the protein expression levels of HSP27 in contrast to TGF- $\beta$. We previously established WT HSP27-overexpressing MC3T3-E1 cells $(22,31)$. In the present study, we investigated the role of HSP27 in the PDGF-BBinduced osteoblast cell migration using the mutant cells. The PDGF-BB-stimulated migrated cell numbers in the HSP27overexpressing cells were markedly less than those in the control cells. We previously demonstrated that HSP27 in the WT HSP27-transfected MC3T3-E1 cells was not phosphorylated (22). However, we found here that PDGF-BB induced the phosphorylation of HSP27 in the WT HSP27-overexpressing MC3T3-E1 cells. Based on our findings, it is probable that HSP27 plays a suppressive role in the PDGF-BB-induced migration of osteoblast-like MC3T3-E1 cells, and that HSP27 in the WT HSP27-expressing cells could be phosphorylated at least in part stimulated by PDGF-BB.

It is currently established that the functions of HSP27 are regulated by post-translational modifications including phosphorylation (10). Regarding the phosphorylation of HSP27, unphosphorylated HSP27 forms aggregated multimers while the phosphorylation of HSP27 results in conformational changes, such as dimers $(12,13)$. During the processes of cell migration, phosphorylated HSP27 reportedly regulates actin filament dynamics in cytoskeleton organization $(38,39)$. It is likely that the phosphorylation of HSP27 stimulated by PDGF-BB itself could affect the PDGF-BB-induced migration of HSP27expressing osteoblasts. Thus, we established two mutant HSP27-transfected osteoblast-like MC3T3-E1 cell lines, 3A and 3D cells, which transiently express the unphosphorylated state and the phospho-mimic state of HSP27, respectively (31), and investigated the effect of HSP27 phosphorylation on the PDGF-BB-induced migration of MC3T3-E1 cells. We demonstrated that the PDGF-BB-stimulated migrated cell numbers of the 3D cells were markedly decreased compared with those of the $3 \mathrm{~A}$ cells. We also showed that the PDGF-BB-induced migrated cell numbers of the WT HSP27-overexpressing cells were less than those of the $3 \mathrm{~A}$ cells. As HSP27 in the WT HSP27-overexpressing cells could be phosphorylated at least in part stimulated by PDGF-BB, our findings suggest that the suppressive effect by HSP27 in its phosphorylated form of the PDGF-BB-stimulated cell migration is greater than in its unphosphorylated form. HSP27 oligomerization is mainly regulated by Ser-78 and/or Ser-82 phosphorylation (13). Therefore, it is probable that we could cover major relevant phosphorylation sites while making $3 \mathrm{~A}$ and $3 \mathrm{D}$ mutants. Based on these findings, it is possible that the conformational change of HSP27 by its phosphorylation affects PDGF-BB-stimulated migration of osteoblast-like MC3T3-E1 cells.

We previously reported that PDGF-BB stimulates IL-6 synthesis at least in part via p44/p42 MAPK, p38 MAPK and SAPK/JNK in osteoblast-like MC3T3-E1 cells (7). Thus, in order to clarify the involvement of these three MAPKs in the PDGF-BB-induced MC3T3-E1 cell migration, we examined the effects of PD98059 (32), SB203580 (33) or SP600125 (34) on the migration. PD98059, SB203580 and SP600125 significantly reduced the PDGF-BB-stimulated migration. Although it is possible that some pharmacological effects other than the specificity may be involved in the suppression, our findings suggest that PDGF-BB induced the migration of osteoblast-like MC3T3-E1 cells through the activation of p44/p42 MAPK, p38 MAPK and SAPK/JNK. On the other hand, in our previous studies $(7,8)$ we demonstrated that PI3K/Akt and p70 S6 kinase limit the PDGF-BB-stimulated IL-6 synthesis in osteoblast-like MC3T3-E1 cells. In order to investigate whether PI3K/Akt or p70 S6 kinase is involved in the PDGF-BB-induced MC3T3-E1 cell migration, we examined the effects of Akt inhibitor, $1_{\mathrm{L}}-6$-hydroxymethyl-chiro-inositol 2-(R)-2-O-methyl-3-O-octadecylcarbonate (35) or rapamycin (36) on the migration. The Akt inhibitor or rapamycin failed to affect the PDGF-BB-stimulated migration of these cells. Thus, it seems unlikely that either PI3K/Akt or p70 S6 kinase regulates the migration stimulated by PDGF-BB in MC3T3-E1 cells. Taking our findings into account, it is most likely that p44/p42 MAPK, p38 MAPK and SAPK/JNK, but not PI3K/Akt or p70 S6 kinase, act as positive regulators in the PDGF-BB-induced migration of osteoblast-like MC3T3-E1 cells.

In order to further investigate the relationship between HSP27 and PDGF signaling in osteoblast-like MC3T3-E1 cells, we examined the effects of PDGF-BB on the phosphorylation of p44/p42 MAPK, p38 MAPK or SAPK/JNK in the WT HSP27 vector-transfected cells compared with those in the control empty vector-transfected cells. However, we did not observe any significant differences in their phosphorylation levels between the WT HSP27-overexpressing cells and the control cells. In addition, we found that there were no significant differences between the $3 \mathrm{~A}$ and $3 \mathrm{D}$ cells in the PDGF-BB-induced phosphorylation levels of p44/p42 MAPK, p38 MAPK or SAPK/JNK. Taking our findings into account, it seems unlikely that HSP27, regardless of its phosphorylation, regulates PDGF-BB-induced osteoblast-like MC3T3-E1 cell migration via activation of p44/p42 MAPK, p38 MAPK or SAPK/JNK. It is possible that HSP27 may act at a point downstream of these molecules or another target, resulting in the downregulation of osteoblast-like MC3T3-E1 cell migration. Our present findings, showing that HSP27 could function as a negative regulator in the PDGF-BB-stimulated migration of osteoblasts, and phosphorylated HSP27 enhances the suppression, provide a new insight concerning small molecular HSP as a bone remodeling modulator. It has been reported that other migratory mediators downstream of PI3K 
such as neuronal Wiskott-Aldrich syndrome protein, Rac and RhoA are involved in the migration induced by PDGF in NIH-3T3 fibroblasts (40). Therefore, it is possible that the mediators described above are involved in the suppression by HSP27 of the PDGF-BB-induced migration in osteoblast-like MC3T3-E1 cells. The Boyden chamber assay adopted here does not distinguish between cells that lose their chemoattraction and those that are no longer changing to a migratory phenotype or dysregulation in adherence. Further investigation including cell adhesion, lamellipodia or filopodia formation would be required to clarify the detailed mechanism underlying the suppressive effect of HSP27 on the migration of osteoblasts.

In conclusion, our results strongly suggest that HSP27 functions as a negative regulator in the PDGF-BB-stimulated migration of osteoblasts and the suppressive effect is amplified by the phosphorylation state of HSP27.

\section{Acknowledgements}

We thank Dr. C. Schafer (Klinikum Grosshadern, Ludwig Maximilians University, Munich, Germany) for providing the mutant HSP27 cDNA and Mrs. Yumiko Kurokawa for her skillful technical assistance. This investigation was supported in part by a Grant-in-Aid for Scientific Research (19591042) from the Ministry of Education, Culture, Sports, Science and Technology of Japan, a Grant-in-Aid for Scientific Research (H25-Aging-General-004) from the Ministry of Health, Labor and Welfare of Japan, and the Research Funding for Longevity Sciences (25-4, 26-12) from the National Center for Geriatrics and Gerontology (NCGG), Japan.

\section{References}

1. Heldin $\mathrm{CH}$ and Westermark B: Mechanism of action and in vivo role of platelet-derived growth factor. Physiol Rev 79: 1283-1316, 1999.

2. Wang Z, Ahmad A, Li Y, Kong D, Azmi AS, Banerjee S and Sarkar FH: Emerging roles of PDGF-D signaling pathway in tumor development and progression. Biochim Biophys Acta 1806: 122-130, 2010.

3. Kular J, Tickner J, Chim SM and Xu J: An overview of the regulation of bone remodelling at the cellular level. Clin Biochem 45: 863-873, 2012.

4. Canalis E: Growth factor control of bone mass. J Cell Biochem 108: 769-777, 2009.

5. Caplan AI and Correa D: PDGF in bone formation and regeneration: new insights into a novel mechanism involving MSCs. J Orthop Res 29: 1795-1803, 2011.

6. Hollinger JO, Hart CE, Hirsch SN, Lynch S and Friedlaender GE: Recombinant human platelet-derived growth factor: biology and clinical applications. J Bone Joint Surg Am 90 (Suppl 1): $48-54,2008$

7. Takai S, Tokuda H, Hanai Y and Kozawa O: Limitation by p70 S6 kinase of platelet-derived growth factor-BB-induced interleukin 6 synthesis in osteoblast-like MC3T3-E1 cells. Metabolism 56: 476-483, 2007.

8. Hanai Y, Tokuda H, Ohta T, Matsushima-Nishiwaki R, Takai S and Kozawa O: Phosphatidylinositol 3-kinase/Akt auto-regulates PDGF-BB-stimulated interleukin-6 synthesis in osteoblasts. J Cell Biochem 99: 1564-1571, 2006.

9. Hengartner NE, Fiedler J, Ignatius A and Brenner RE: IL-1 $\beta$ inhibits human osteoblast migration. Mol Med 19: 36-42, 2013.

10. Mymrikov EV, Seit-Nebi AS and Gusev NB: Large potentials of small heat shock proteins. Physiol Rev 91: 1123-1159, 2011.

11. Bakthisaran R, Tangirala R and Rao ChM: Small heat shock proteins: role in cellular functions and pathology. Biochim Biophys Acta 1854: 291-319, 2015.
12. Kostenko $S$ and Moens U: Heat shock protein 27 phosphorylation: kinases, phosphatases, functions and pathology. Cell Mol Life Sci 66: 3289-3307, 2009.

13. Katsogiannou M, Andrieu C and Rocchi P: Heat shock protein 27 phosphorylation state is associated with cancer progression. Front Genet 5: 346, 2014.

14. Leonardi R, Barbato E, Paganelli $\mathrm{C}$ and Lo Muzio L: Immunolocalization of heat shock protein 27 in developing jaw bones and tooth germs of human fetuses. Calcif Tissue Int 75: 509-516, 2004.

15. Shakoori AR, Oberdorf AM, Owen TA, Weber LA, Hickey E, Stein JL, Lian JB and Stein GS: Expression of heat shock genes during differentiation of mammalian osteoblasts and promyelocytic leukemia cells. J Cell Biochem 48: 277-287, 1992.

16. Kozawa O, Niwa M, Matsuno H, Ishisaki A, Kato K and Uematsu T: Stimulatory effect of basic fibroblast growth factor on induction of heat shock protein 27 in osteoblasts: role of protein kinase C. Arch Biochem Biophys 388: 237-242, 2001.

17. Kozawa O, Otsuka T, Hatakeyama D, Niwa M, Matsuno H, Ito H, Kato K, Matsui N and Uematsu T: Mechanism of prostaglandin $\mathrm{D}_{2}$-stimulated heat shock protein 27 induction in osteoblasts. Cell Signal 13: 535-541, 2001.

18. Hatakeyama D, Kozawa O, Niwa M, Matsuno H, Kato K, Tatematsu N, Shibata T and Uematsu T: Inhibition by adenylyl cyclase-cAMP system of ET-1-induced HSP27 in osteoblasts. Am J Physiol Endocrinol Metab 281: E1260-E1266, 2001.

19. Tokuda H, Kozawa O, Niwa M, Matsuno H, Kato $\mathrm{K}$ and Uematsu T: Mechanism of prostaglandin $\mathrm{E}_{2}$-stimulated heat shock protein 27 induction in osteoblast-like MC3T3-E1 cells. J Endocrinol 172: 271-281, 2002.

20. Tokuda H, Niwa M, Ishisaki A, Nakajima K, Ito H, Kato K and Kozawa O: Involvement of stress-activated protein kinase (SAPK)/c-Jun N-terminal kinase (JNK) in prostaglandin $\mathrm{F}_{2 a}$-induced heat shock protein 27 in osteoblasts. Prostaglandins Leukot Essent Fatty Acids 70: 441-447, 2004.

21. Hayashi K, Takai S, Matsushima-Nishiwaki R, Hanai Y, Kato K, Tokuda $\mathrm{H}$ and Kozawa O: (-)-Epigallocatechin gallate reduces transforming growth factor $\beta$-stimulated HSP27 induction through the suppression of stress-activated protein kinase/c-Jun N-terminal kinase in osteoblasts. Life Sci 82: 1012-1017, 2008.

22. Kato K, Adachi S, Matsushima-Nishiwaki R, Minamitani C, Natsume H, Katagiri Y, Hirose Y, Mizutani J, Tokuda H, Kozawa O, et al: Regulation by heat shock protein 27 of osteocalcin synthesis in osteoblasts. Endocrinology 152: 1872-1882, 2011.

23. Kubisch C, Dimagno MJ, Tietz AB, Welsh MJ, Ernst SA, Brandt-Nedelev B, Diebold J, Wagner AC, Göke B, Williams JA, et al: Overexpression of heat shock protein Hsp27 protects against cerulein-induced pancreatitis. Gastroenterology 127: 275-286, 2004.

24. Sudo H, Kodama HA, Amagai Y, Yamamoto S and Kasai S: In vitro differentiation and calcification in a new clonal osteogenic cell line derived from newborn mouse calvaria. J Cell Biol 96: 191-198, 1983.

25. Kozawa O, Tokuda H, Miwa M, Kotoyori J and Oiso Y: Cross-talk regulation between cyclic AMP production and phosphoinositide hydrolysis induced by prostaglandin $\mathrm{E}_{2}$ in osteoblast-like cells. Exp Cell Res 198: 130-134, 1992.

26. Karagiosis SA, Chrisler WB, Bollinger N and Karin NJ: Lysophosphatidic acid-induced ERK activation and chemotaxis in MC3T3-E1 preosteoblasts are independent of EGF receptor transactivation. J Cell Physiol 219: 716-723, 2009.

27. Laemmli UK: Cleavage of structural proteins during the assembly of the head of bacteriophage T4. Nature 227: 680-685, 1970.

28. Kato $\mathrm{K}$, Ito $\mathrm{H}$, Hasegawa $\mathrm{K}$, Inaguma $\mathrm{Y}$, Kozawa $\mathrm{O}$ and Asano T: Modulation of the stress-induced synthesis of hsp27 and $\alpha \mathrm{B}$-crystallin by cyclic AMP in C6 rat glioma cells. J Neurochem 66: 946-950, 1996.

29. Kozawa O, Niwa M, Matsuno H, Tokuda H, Miwa M, Ito H, Kato K and Uematsu T: Sphingosine 1-phosphate induces heat shock protein 27 via p38 mitogen-activated protein kinase activation in osteoblasts. J Bone Miner Res 14: 1761-1767, 1999.

30. Hatakeyama D, Kozawa O, Niwa M, Matsuno H, Ito H, Kato K, Tatematsu N, Shibata T and Uematsu T: Upregulation by retinoic acid of transforming growth factor- $\beta$-stimulated heat shock protein 27 induction in osteoblasts: involvement of mitogen-activated protein kinases. Biochim Biophys Acta 1589: 15-30, 2002.

31. KuroyanagiG,TokudaH, YamamotoN,Matsushima-NishiwakiR, Kozawa O and Otsuka T: Unphosphorylated HSP27 (HSPB1) regulates the translation initiation process via a direct association with eIF4E in osteoblasts. Int J Mol Med 36: 881-889, 2015. 
32. Alessi DR, Cuenda A, Cohen P, Dudley DT and Saltiel AR: PD 098059 is a specific inhibitor of the activation of mitogen-activated protein kinase kinase in vitro and in vivo. $\mathrm{J}$ Biol Chem 270: 27489-27494, 1995.

33. Cuenda A, Rouse J, Doza YN, Meier R, Cohen P, Gallagher TF, Young PR and Lee JC: SB 203580 is a specific inhibitor of a MAP kinase homologue which is stimulated by cellular stresses and interleukin-1. FEBS Lett 364: 229-233, 1995.

34. Bennett BL, Sasaki DT, Murray BW, O'Leary EC, Sakata ST, $\mathrm{Xu}$ W, Leisten JC, Motiwala A, Pierce S, Satoh Y, et al: SP600125, an anthrapyrazolone inhibitor of Jun N-terminal kinase. Proc Natl Acad Sci USA 98: 13681-13686, 2001.

35. Hu Y, Qiao L, Wang S, Rong SB, Meuillet EJ, Berggren M, Gallegos A, Powis G and Kozikowski AP: 3-(Hydroxymethyl)-bearing phosphatidylinositol ether lipid analogues and carbonate surrogates block PI3-K, Akt, and cancer cell growth. J Med Chem 43: 3045-3051, 2000.

36. Price DJ, Grove JR, Calvo V, Avruch J and Bierer BE: Rapamycin-induced inhibition of the 70-kilodalton S6 protein kinase. Science 257: 973-977, 1992.
37. Chun KH, Kosmeder JW II, Sun S, Pezzuto JM, Lotan R, Hong WK and Lee HY: Effects of deguelin on the phosphatidylinositol 3-kinase/Akt pathway and apoptosis in premalignant human bronchial epithelial cells. J Natl Cancer Inst 95: 291-302, 2003

38. Clarke JP and Mearow KM: Cell stress promotes the association of phosphorylated HspB1 with F-actin. PLoS One 8: e68978, 2013.

39. Lavoie JN, Hickey E, Weber LA and Landry J: Modulation of actin microfilament dynamics and fluid phase pinocytosis by phosphorylation of heat shock protein 27. J Biol Chem 268: 24210-24214, 1993.

40. Jiménez C, Portela RA, Mellado M, Rodríguez-Frade JM, Collard J, Serrano A, Martínez-A C, Avila J and Carrera AC: Role of the PI3K regulatory subunit in the control of actin organization and cell migration. J Cell Biol 151: 249-262, 2000. 